This item was submitted to Loughborough's Research Repository by the author.

Items in Figshare are protected by copyright, with all rights reserved, unless otherwise indicated.

\title{
Buffer-aided relay selection with reduced packet delay in cooperative networks
}

PLEASE CITE THE PUBLISHED VERSION

http://dx.doi.org/10.1109/TVT.2016.2573378

PUBLISHER

IEEE

VERSION

VoR (Version of Record)

PUBLISHER STATEMENT

This work is made available according to the conditions of the Creative Commons Attribution 3.0 Unported (CC BY 3.0) licence. Full details of this licence are available at: http://creativecommons.org/licenses/by/3.0/

\section{LICENCE}

CC BY 3.0

\section{REPOSITORY RECORD}

Tian, Zhao, Yu Gong, Gaojie Chen, and Jonathon Chambers. 2016. "Buffer-aided Relay Selection with Reduced Packet Delay in Cooperative Networks". Loughborough University.

https://hdl.handle.net/2134/22250. 


\title{
Buffer-Aided Relay Selection With Reduced Packet Delay in Cooperative Networks
}

\author{
Zhao Tian, Member, IEEE, Yu Gong, Gaojie Chen, Member, IEEE, and Jonathon A. Chambers, Fellow, IEEE
}

\begin{abstract}
Applying data buffers at relay nodes significantly improves the outage performance in relay networks, but the performance gain is often at the price of long packet delays. In this paper, a novel relay selection scheme with significantly reduced packet delay is proposed. The outage probability and average packet delay of the proposed scheme under different channel scenarios are analyzed. Simulation results are also given to verify the analysis. The analytical and simulation results show that, compared with non-buffer-aided relay selection schemes, the proposed scheme has not only significant gain in outage performance but also similar average packet delay when the channel signal-to-noise ratio (SNR) is high enough, making it an attractive scheme in practice.
\end{abstract}

Index Terms-Average delay, buffer-aided relay, relay selection.

\section{INTRODUCTION}

$\mathbf{R}$ ELAY selection provides an attractive way to harvest the diversity gain in multiple relay cooperative networks [1], [2]. A typical relay selection system is shown in Fig. 1, which includes one source node $(S)$, one destination node $(D)$, and $N$ relay nodes $\left(R_{k}, 1 \leq k \leq N\right)$. Analysis shows that full diversity order can be achieved with the best selected relay [3]-[5]. In the traditional max-min relay selection scheme, the best relay is selected with the highest gain among all of the minima of the source-to-relay and relay-to-destination channel gain pairs [6]. While the max-min scheme achieves a diversity order of $N$, its performance is practically limited by the constraint that the best source-to-relay and relay-to-destination links for a packet transmission must be determined concurrently. Recent research has, on the other hand, found that introducing data buffers at the relays yields significant performance advantage in practical systems [7]-[10]. Buffer-aided relays have also been used in ap-

Manuscript received March 2, 2016; accepted May 8, 2016. Date of publication May 30, 2016; date of current version March 10, 2017. This work was supported in part by the Engineering and Physical Sciences Research Council under Grant EP/K014307/2 and in part by the Ministry of Defence's University Defence Research Collaboration in Signal Processing. The review of this paper was coordinated by Prof. R. Jäntti. (Corresponding author: Gaojie Chen.)

Z. Tian is with School of Engineering and Applied Science, Aston University, Birmingham B4 7ET, U.K. (e-mail: z.tian@lboro.ac.uk).

Y. Gong is with the Advanced Signal Processing Group, Loughborough University, Loughborough LE11 3TU, U.K. (e-mail: y.gong@lboro.ac.uk).

G. Chen is with the Department of Engineering Science, University of Oxford, Oxford OX1 3PJ, U.K. (e-mail: gaojie.chen@eng.ox.ac.uk).

J. A. Chambers is with the Communications, Sensors, Signal and Information Processing Group, Newcastle University, Newcastle upon Tyne NE1 7RU, U.K. (e-mail: jonathon.chambers@ncl.ac.uk).

Color versions of one or more of the figures in this paper are available online at http://ieeexplore.ieee.org.

Digital Object Identifier 10.1109/TVT.2016.2573378

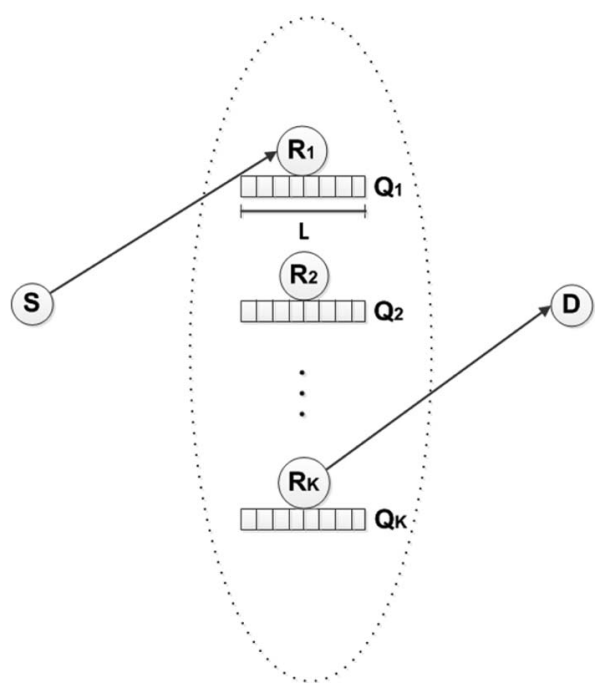

Fig. 1. System model of the relay selection system.

plications including adaptive link selection [11], [12], cognitive radio networks [13], and physical layer network security [14].

Typical buffer-aided relay selection schemes include the max-max [7] and max-link [8] schemes. In max-max relay selection, at one time slot $t$, the best link among all sourceto-relay channels is selected, and a data packet is sent to the selected relay and stored in the buffer. At the next time slot $(t+1)$, the best link among all relay-to-destination channels is selected, and the selected relay (which is often not the same relay selected at time $t$ ) forwards one data packet from its buffer to the destination. The max-max scheme has significant coding gain over the traditional max-min scheme. In the max-link scheme [8], the best link is selected among all available sourceto-relay and relay-to-destination links. Depending on whether a source-to-relay or a relay-to-destination link is selected, either the source transmits a packet to the selected relay or the selected relay forwards a stored packet to the destination. As a result, the max-link relay selection not only has coding gain over the max-min scheme but also has higher diversity order than both the max-min and max-max schemes, making it more attractive than its max-max counterpart.

The performance gain of either the buffer-aided max-max or max-link schemes is, however, at the price of much increased packet delay. In the non-buffer-aided relay selection scheme (e.g., the max-min scheme), it always takes two time slots for every packet passing through the network, corresponding to the source-to-relay and relay-to-destination transmission, respectively. In the buffer-aided approach, in contrast, when a packet is transmitted to a relay node, it is stored in the 
buffer and will not be forwarded to the destination until the corresponding relay-to-destination link is selected. As a result, different packets in the buffer-aided relay network may endure different delays. To be specific, in either the max-max or maxlink scheme, the average packet delay increases linearly with relay number and buffer size. On the other hand, to achieve high performance gain, the relay number and the buffer size in the max-max or max-min scheme are often set as high as possible. This makes the existing buffer-aided relay selection schemes unsuitable in most applications, particularly in 5G mobile systems which require ultralow latency.

While packet delay reduction has been investigated in adaptive link selection with infinite buffer size (e.g., [11]), little has been done for buffer-aided relay selection with finite buffer size. In this paper, we propose a novel buffer-aided relay selection scheme with significantly reduced packet delay. This is achieved by giving higher priority to select the relay-todestination than the source-to-relay links, so that the data queues at relay buffers are as short as possible. The main contributions of this paper are listed as follows.

- Proposing a novel relay selection scheme. The proposed scheme provides a simple yet effective way to reduce the packet delay in the buffer-aided relay selection.

- Deriving the closed-form expression for outage probability. The analysis is based on the general asymmetric channel assumption that the source-to-relay and relay-todestination links may have different average gains.

- Obtaining the closed-form expression for the average packet delay. Using Little's law, the average packet delay of the proposed scheme is analytically obtained.

- Analyzing the asymptotic performance that the channel SNR goes to infinity. The asymptotic performances, including diversity order, coding gain, and average packet delay for infinite channel SNR, are analyzed.

The remainder of this paper is organized as follows: Section II proposes the new relay selection scheme; Section III analyzes the outage probability; Section IV analyzes the average packet delay; Section V analyzes the asymptotic performance; Section VI shows simulation results; and Section VII concludes this paper.

\section{BUFFER-AIDED RELAY SELECTION WITH REDUCED DELAY}

The system model of buffer-aided relay selection is similar to that shown in Fig. 1, except that every relay is equipped with a data buffer $Q_{k}(1 \leq k \leq N)$ of finite size $L$. We assume that relays apply the decode-and-forward (DF) protocol. The channel coefficients for $S \rightarrow R_{k}$ and $R_{k} \rightarrow D$ links at time slot $t$ are denoted by $h_{s r_{k}}(t)$ and $h_{r_{k} d}(t)$, respectively. All channels are Rayleigh fading, and the average channel gains for $S \rightarrow R_{k}$ and $R_{k} \rightarrow D$ links are given by

$$
\bar{\gamma}_{s r}=E\left[\left|h_{s r_{k}}(t)\right|^{2}\right], \quad \bar{\gamma}_{r d}=E\left[\left|h_{r_{k} d}(t)\right|^{2}\right], \quad \text { for all } k
$$

respectively. We assume without losing generality that all transmission power and noise variances are normalized to unity. We also assume that channel gains in either the source-to-relay or relay-to-destination links are independent and identically distributed (i.i.d.), but in general $\bar{\gamma}_{s r} \neq \bar{\gamma}_{r d}$.

In the existing buffer-aided max-max and max-min relay selection schemes, the average packet delay increases linearly with relay number and buffer size. The large delay is due to the packets queuing at the buffers. This can be seen, for example, in the max-link scheme with relay number $N$ and buffer size $L>2$. Specifically, we assume that all buffers are initially empty and a packet $s_{1}$ is sent to relay $R_{1}$ at time $t=1$. Then, at the next time $t=2$, except for $R_{1}$ which contains $s_{1}$, all other buffers are still empty. Thus, there are $(N+1)$ available links for selection in total: $N$ from source-to-relay $\left(S \rightarrow R_{k}\right.$ for all $k$ ) links and one from relay-to-destination $\left(R_{1} \rightarrow D\right)$ link. Because the max-link scheme always selects the strongest link among all available links, the probability that $R_{1} \rightarrow D$ is selected and $s_{1}$ is forwarded to the destination is $\left.1 /(N+1)\right)$. In other words, it is more likely [with the probability of $N /(N+$ 1)] that $s_{1}$ remains in $R_{1}$ at $t=2$, leading to one extra time slot in packet delay. It is clear that this extra delay may be avoided by forwarding $s_{1}$ to the destination immediately at $t=2$, once the corresponding $R_{1} \rightarrow D$ link is not in outage, even if it is not the strongest link.

This leads to a new principle of buffer-aided relay selection, i.e., to transmit the packets already in the buffers as fast as possible. This translates into giving higher priority to select the relay-to-destination links: only when no relay-to-destination link can be selected are the source-to-relay links considered. As a result, the packet queuing lengths at the relay buffers are minimized, as well as the average packet delay.

To be specific, at time slot $t$, the link selection rule is described as follows.

1) Choose the link with the highest channel SNR among all available relay-to-destination links $\left(\left|h_{r_{k} d}(t)\right|^{2}\right)$. If the chosen link is not in outage, the corresponding relay forwards a packet from its buffer to the destination.

2) Otherwise, if the selected link in step 1) is in outage or there are no available relay-to-destination links at time $t$, choose the link with the highest channel SNR among all available source-to-relay links $\left(\left|h_{s r_{k}}(t)\right|^{2}\right)$. If the selected link is not in outage, the source transmits one packet to the corresponding relay and the packet is stored in the buffer. Otherwise, outage occurs.

The aforementioned proposed scheme is easy to implement because it requires the same knowledge as that in the existing buffer-aided max-max or max-min scheme. In the following two sections, the outage and delay performance of the proposed scheme will be analyzed, respectively.

\section{Outage Probability}

The numbers of data packets in all of the relay buffers form a "state." With $N$ relays and buffer size $L$, there are $(L+1)^{N}$ states in total. The $l$ th state vector is defined as

$$
\mathbf{s}_{l}=\left[\Psi_{l}\left(Q_{1}\right), \ldots, \Psi_{l}\left(Q_{K}\right)\right], \quad l=1, \ldots,(L+1)^{N}
$$

where $\Psi_{l}\left(Q_{k}\right)$ gives the number of data packets in buffer $Q_{k}$ at state $s_{l}$. It is clear that $0 \leq \Psi_{l}\left(Q_{k}\right) \leq L$. 
Every state corresponds to one pair of $\left(K_{s_{l}}^{S \rightarrow R}, K_{s_{l}}^{R \rightarrow D}\right)$, corresponding to the numbers of available source-to-relay and relay-to-destination links, respectively. A source-to-relay link is considered available when the buffer of the corresponding relay node is not full, and a relay-to-destination link is available when the corresponding relay buffer is not empty. At state $s_{l}$, the total numbers of available source-to-relay and relay-to-destination links are denoted by $K_{s_{l}}^{S \rightarrow R}$ and $K_{s_{l}}^{R \rightarrow D}$, respectively. It is clear that $0 \leq K_{s_{l}}^{S \rightarrow R} \leq N$ and $0 \leq K_{s_{l}}^{R \rightarrow D} \leq N$. Specifically, if none of the buffers are full or empty, all links are available, such that $K_{s_{l}}^{S \rightarrow R}=K_{s_{l}}^{R \rightarrow D}=N$.

Considering all possible states, the outage probability of the proposed buffer-aided scheme can be obtained as

$$
P_{\text {out }}=\sum_{l=1}^{(L+1)^{N}} \pi_{l} \cdot p_{\text {out }}^{s_{l}}
$$

where $\pi_{l}$ is the stationary probability for state $\mathbf{s}_{l}$, and $p_{\text {out }}^{s_{l}}$ is the outage probability at state $\mathbf{s}_{l}$. In the following two subsections, we derive $p_{\text {out }}^{s_{l}}$ and $\pi_{l}$, respectively.

\section{A. $p_{\text {out }}^{s_{l}}:$ Outage Probability at State $\mathbf{s}_{l}$}

For independent Rayleigh fading channels, the instantaneous SNR for every channel, i.e., $\gamma_{w}\left(w \in\left\{s r_{k}, r_{k} d\right\}\right)$, is independently exponentially distributed. In the proposed scheme, outage occurs if all available source-to-relay links and relay-todestination links are in outage. Thus, the outage probability at state $\mathbf{s}_{l}$ is given by

$$
p_{\text {out }}^{s_{l}}=p_{\text {out }}^{S \rightarrow R} \cdot p_{\text {out }}^{R \rightarrow D}
$$

where

$$
\begin{aligned}
& p_{\text {out }}^{S \rightarrow R}=\left(1-e^{-\frac{\Delta}{\gamma_{s r}}}\right)^{K_{s_{l}}^{S \rightarrow R}} \\
& p_{\text {out }}^{R \rightarrow D}=\left(1-e^{-\frac{\Delta}{\gamma_{r d}}}\right)^{K_{s_{l}}^{R \rightarrow D}}
\end{aligned}
$$

where $p_{\text {out }}^{S \rightarrow R}$ and $p_{\text {out }}^{R \rightarrow D}$ are the probabilities that all available source-to-relay links and relay-to-destination links are in outage, respectively; $r_{t}$ is the target data rate; and $\Delta=2^{r_{t}}-1$.

\section{B. $\pi_{l}$ : Stationary Probability of the State $s_{l}$}

We denote $\mathbf{A}$ as the $(L+1)^{N} \times(L+1)^{N}$ state transition matrix, where the entry $\mathbf{A}_{n, l}=P\left(X_{t+1}=s_{n} \mid X_{t}=s_{l}\right)$ is the transition probability that the state moves from $s_{l}$ at time $t$ to $s_{n}$ at time $(t+1)$.

We assume that, at time slot $t$, the state is at $s_{l}$. The probability to select one relay-to-destination link when not all of the available relay-to-destination links are in outage is given by

$$
\begin{aligned}
p_{s_{l}}^{R \rightarrow D} & =\frac{1}{K_{s_{l}}^{R \rightarrow D}} \cdot\left(1-p_{\text {out }}^{R \rightarrow D}\right) \\
& =\frac{1}{K_{s_{l}}^{R \rightarrow D}} \cdot\left(1-\left(1-e^{-\frac{\Delta}{\gamma_{r d}}}\right)^{K_{s_{l}}^{R \rightarrow D}}\right) .
\end{aligned}
$$

On the other hand, because a source-to-relay link is selected only when all relay-to-destination links are in outage and not all source-to-relay links are in outage, the probability to select one source-to-relay link at state $s_{l}$ is given by

$$
\begin{aligned}
& p_{s_{l}}^{S \rightarrow R} \\
& =\frac{1}{K_{s_{l}}^{S \rightarrow R}} \cdot p_{\mathrm{out}}^{R \rightarrow D} \cdot\left(1-p_{\mathrm{out}}^{S \rightarrow R}\right) \\
& =\frac{1}{K_{s_{l}}^{S \rightarrow R}} \cdot\left(1-e^{-\frac{\Delta}{\gamma_{r d}}}\right)^{K_{s_{l}}^{R \rightarrow D}} \cdot\left(1-\left(1-e^{-\frac{\Delta}{\gamma_{s r}}}\right)^{K_{s_{l}}^{S \rightarrow R}}\right) .
\end{aligned}
$$

With these observations, the $(n, l)$ th entry of the state transition matrix $\mathbf{A}$ is expressed as

$$
\mathbf{A}_{n, l}=\left\{\begin{array}{lll}
p_{\text {out }}^{s_{l}}, & \text { if } \quad s_{n}=s_{l} \\
p_{s_{l}}^{R \rightarrow D} & \text { if } \quad s_{n} \in U_{s_{l}}^{R \rightarrow D} \\
p_{s_{l}}^{S \rightarrow R}, & \text { if } \quad s_{n} \in U_{s_{l}}^{S \rightarrow R} \\
0, & \text { elsewhere }
\end{array}\right.
$$

where $p_{\text {out }}^{s_{l}}, p_{s_{l}}^{R \rightarrow D}$, and $p_{s_{l}}^{S \rightarrow R}$ are given by (4), (6), and (7), respectively; $U_{s_{l}}^{R \rightarrow D}$ and $U_{s_{l}}^{S \rightarrow R}$ are the sets containing all states to which $s_{l}$ can move when a relay-to-destination link or a source-to-relay link is selected, respectively.

Because the transition matrix $\mathbf{A}$ in (8) is column stochastic, irreducible, and aperiodic, ${ }^{1}$ the stationary state probability vector is obtained as (see [15])

$$
\boldsymbol{\pi}=(\mathbf{A}-\mathbf{I}+\mathbf{B})^{-1} \mathbf{b}
$$

where $\boldsymbol{\pi}=\left[\pi_{1}, \ldots, \pi_{(L+1)^{N}}\right]^{T}, \mathbf{b}=(1,1, \ldots, 1)^{T}, \mathbf{I}$ is the identity matrix, and $\mathbf{B}_{n, l}$ is an $n \times l$ all-one matrix.

Finally, substituting (8) and (9) into (3) gives the outage probability as

$$
\begin{aligned}
P_{\text {out }} & =\sum_{l=1}^{(L+1)^{N}} \pi_{l} \cdot p_{\text {out }}^{s_{l}}=\operatorname{diag}(\mathbf{A}) \cdot \boldsymbol{\pi} \\
& =\operatorname{diag}(\mathbf{A}) \cdot(\mathbf{A}-\mathbf{I}+\mathbf{B})^{-1} \mathbf{b}
\end{aligned}
$$

where $\operatorname{diag}(\mathbf{A})$ is a vector consisting of all diagonal elements of $\mathbf{A}$.

\section{Average Packet Delay}

The delay of a packet in the system is the duration between the time when the packet leaves the source node and the time when it arrives the destination. Because it takes one time slot to transmit a packet from the source to a relay node, the average packet delay in the system is given by

$$
\bar{D}=1+\bar{D}_{r}
$$

where $\bar{D}_{r}$ is the average delay at the relay nodes.

Because the average delay through every relay node is the same, only the average delay through relay $R_{k}$ is analyzed in

\footnotetext{
${ }^{1}$ Column stochastic means that all entries in any column sum up to one, irreducible means that it is possible to move from any state to any state, and aperiodic means that it is possible to return to the same state at any steps [15].
} 
the following. Based on Little's law [16], the average packet delay at relay $R_{k}$ is given by

$$
\bar{D}_{r}=\bar{D}_{k}=\frac{\bar{L}_{k}}{\bar{\eta}_{k}}
$$

where $\bar{L}_{k}$ and $\bar{\eta}_{l}$ are the average queuing length and average throughput at $R_{k}$, respectively.

The average queuing length at $R_{k}$ is obtained by averaging the queuing lengths at buffer $Q_{k}$ over all states, or

$$
\bar{L}_{k}=\sum_{l=1}^{(L+1)^{N}} \pi_{l} \Psi_{l}\left(Q_{k}\right)
$$

where $\Psi_{l}\left(Q_{k}\right)$ gives the number of packets (or the buffer length) of buffer $Q_{k}$ at state $s_{l}$, and $\pi_{l}$ is given by (9).

On the other hand, because the probabilities to select any of the relays are the same, the average throughput at relay $R_{k}$ is given by

$$
\bar{\eta}_{k}=\frac{\bar{\eta}}{N}
$$

where $\bar{\eta}$ is the average throughput of the overall system network. For delay-limited transmission, the average throughput $\bar{\eta}$ is obtained as (see [17] and [18])

$$
\bar{\eta}=R \cdot\left(1-P_{\text {out }}\right)
$$

where $R$ is the average data rate of the system (without considering the outage probability). In the proposed scheme, every packet requires two time slots (not necessarily consecutively) to reach the destination, we have $R=1 / 2$, and thus

$$
\bar{\eta}_{k}=\frac{1-P_{\text {out }}}{2 N} .
$$

Substituting (13) and (16) into (12), and further into (11), gives

$$
\bar{D}=1+\frac{2 \cdot N \cdot \sum_{l=1}^{(L+1)^{N}} \pi_{l} \Psi_{l}\left(Q_{k}\right)}{1-P_{\text {out }}} .
$$

\section{ASYMPtotic PERformance}

This section analyzes the asymptotic performance of the proposed scheme when the average channel SNR goes to infinity. The average channel SNRs for source-to-relay and relay-todestination link can be respectively expressed as

$$
\bar{\gamma}_{s r}=\alpha \bar{\gamma} \quad \text { and } \quad \bar{\gamma}_{r d}=\beta \bar{\gamma}
$$

where $\alpha$ and $\beta$ are positive real constants, and $\bar{\gamma}$ is the normalized average channel SNR. In the following, we first derive the asymptotic outage probability for $\bar{\gamma} \rightarrow \infty$, from which the diversity order, coding gain, and average packet delay are obtained.

\section{A. Asymptotic Outage Probability}

When $\bar{\gamma} \rightarrow \infty$, it is clear from (6) that

$$
\lim _{\bar{\gamma} \rightarrow \infty} p_{s_{l}}^{R \rightarrow D}=1, \quad \text { if } K_{s_{l}}^{R \rightarrow D} \neq 0 .
$$

This implies that any packets in the relay buffers will be forwarded to the destination and a new packet is transmitted to one of the relays only after all buffers are empty. Thus, when $\bar{\gamma} \rightarrow \infty$, the buffers can only be in two possible states, namely, $S^{(0)}$ and $S^{(1)}$, corresponding to the cases that all buffers are empty and only one of the buffers has one packet, respectively. It is then from (3) that

$$
\lim _{\bar{\gamma} \rightarrow \infty} P_{\text {out }}=P\left(S^{(0)}\right) \cdot p_{\text {out }}^{S^{(0)}}+P\left(S^{(1)}\right) \cdot p_{\text {out }}^{S^{(1)}}
$$

where $P\left(S^{(0)}\right)$ and $P\left(S^{(1)}\right)$ are the probabilities that buffers are in states $S^{(0)}$ and $S^{(1)}$, respectively, and $p_{\text {out }}^{S^{(0)}}$ and $p_{\text {out }}^{S^{(1)}}$ are the corresponding outage probabilities.

Suppose that, at time $t$, all buffers are empty, so that the state is in $S^{(0)}$. Then, one packet will be transmitted to a relay at time $(t+1)$, and the state moves to $S^{(1)}$. From (19), the packet in the buffer must be forwarded to the destination at $(t+2)$, and the state returns to $S^{(0)}$. This process continues until all packets are transmitted. Thus, we have

$$
P\left(S^{(0)}\right)=P\left(S^{(1)}\right)=\frac{1}{2} .
$$

When the buffers are in state $S^{(0)}$, there are $N$ available sourceto-relay links and no available relay-to-destination links, or we have

$$
p_{\text {out }}^{S^{(0)}}=\left(1-e^{-\frac{\Delta}{\gamma_{s r}}}\right)^{N} .
$$

When the buffers are in state $S^{(1)}$, there is one available relayto-destination link. In addition, the number of available sourceto-relay links is denoted by $K_{\infty}$, where $K_{\infty}=N-1$ or $N$, for buffer size $L=1$ or larger, respectively. Then, we have

$$
p_{\text {out }}^{S^{(1)}}=\left(1-e^{-\frac{\Delta}{\bar{\gamma}_{s r}}}\right)^{K_{\infty}} \cdot\left(1-e^{-\frac{\Delta}{\bar{\gamma}_{r d}}}\right) .
$$

Substituting (21), (22), and (23) into (20) gives

$$
\begin{aligned}
\lim _{\bar{\gamma} \rightarrow \infty} P_{\text {out }}=\frac{1}{2} \cdot\left(1-e^{-\frac{\Delta}{\alpha \bar{\gamma}}}\right)^{N}+\frac{1}{2} \cdot( & \left.1-e^{-\frac{\Delta}{\alpha \bar{\gamma}}}\right)^{K_{\infty}} \\
& \times\left(1-e^{-\frac{\Delta}{\beta \bar{\gamma}}}\right) .
\end{aligned}
$$

\section{B. Diversity Order}

The diversity order can be defined as

$$
d=-\lim _{\bar{\gamma} \rightarrow \infty} \frac{\log P_{\text {out }}}{\log \bar{\gamma}} .
$$

If the buffer size $L=1$, substituting (24) into (25), and further noting that $e^{x} \approx 1+x$ for very small $x$, we have the diversity order for $L=1$ as

$$
d^{(L=1)}=-\lim _{\bar{\gamma} \rightarrow \infty} \frac{\log \left[\frac{1}{2} \cdot\left(\frac{\Delta}{\alpha \bar{\gamma}}\right)^{N-1} \cdot\left(\frac{\Delta}{\alpha \bar{\gamma}}+\frac{\Delta}{\beta \bar{\gamma}}\right)\right]}{\log \bar{\gamma}}=N .
$$

If the buffer size $L \geq 2$, from (24), the asymptotic outage probability is given by

$$
\lim _{\bar{\gamma} \rightarrow \infty} P_{\text {out }}^{(L \geq 2)}=\lim _{\bar{\gamma} \rightarrow \infty}\left[\frac{1}{2} \cdot\left(\frac{\Delta}{\alpha \bar{\gamma}}\right)^{N} \cdot\left(\frac{\beta \bar{\gamma}+\Delta}{\beta \bar{\gamma}}\right)\right] .
$$

Because

$$
\lim _{\bar{\gamma} \rightarrow \infty}(\beta \bar{\gamma})<\lim _{\bar{\gamma} \rightarrow \infty}(\beta \bar{\gamma}+\Delta)<\lim _{\bar{\gamma} \rightarrow \infty}(2 \cdot \beta \bar{\gamma})
$$

the diversity order for $L \geq 2$ can be obtained as

$$
N<d^{(L \geq 2)}<N+1 .
$$




\section{Coding Gain}

The coding gain is defined as the SNR difference (in decibels) between the traditional max-min and proposed schemes to achieve the same outage probability, or

$$
C(\mathrm{~dB})=-\frac{\lim _{\bar{\gamma} \rightarrow \infty} \Delta_{P}(\bar{\gamma})}{d}
$$

where $d=N$, which is the diversity order, and

$$
\Delta_{P}(\bar{\gamma})=10 \log P_{\text {out }}^{(\max -\min )}(\bar{\gamma})-10 \log P_{\text {out }}^{(L=1)}(\bar{\gamma})
$$

where $P_{\text {out }}^{(\max -\min )}(\bar{\gamma})$ and $P_{\text {out }}^{(L=1)}(\bar{\gamma})$ are the outage probabilities at $\bar{\gamma}$ for the max-min and proposed schemes, respectively. For fair comparison, the buffer size is set as $L=1$, so that the diversity order for the max-min and proposed schemes are the same as $d=N$.

From (24), we have

$$
\begin{aligned}
\lim _{\bar{\gamma} \rightarrow \infty} 10 \log P_{\text {out }}^{(L=1)}=10 \cdot \log & {\left[\frac{1}{2} \cdot\left(\frac{1}{\alpha}\right)^{N-1}\left(\frac{1}{\alpha}+\frac{1}{\beta}\right)\right] } \\
& +\lim _{\bar{\gamma} \rightarrow \infty} 10 \cdot \log \left(\frac{\Delta}{\bar{\gamma}}\right)^{N} .
\end{aligned}
$$

For the traditional max-min scheme, we have

$$
\begin{aligned}
& \lim _{\bar{\gamma} \rightarrow \infty} 10 \log P_{\text {out }}^{(\max -\min )} \\
& \quad=\lim _{\bar{\gamma} \rightarrow \infty} 10 \cdot \log \left(\frac{\Delta}{\alpha \bar{\gamma}}+\frac{\Delta}{\beta \bar{\gamma}}\right)^{N} \\
& \quad=10 \cdot \log \left(\frac{1}{\alpha}+\frac{1}{\beta}\right)^{N}+\lim _{\bar{\gamma} \rightarrow \infty} 10 \cdot \log \left(\frac{\Delta}{\bar{\gamma}}\right)^{N} .
\end{aligned}
$$

Substituting (32) and (33) into (31) gives

$$
\lim _{\bar{\gamma} \rightarrow \infty} \Delta_{P}(\bar{\gamma})=-10 \cdot \log \left[\frac{1}{2}\left(\frac{\beta}{\alpha+\beta}\right)^{N-1}\right] .
$$

Finally, substituting (34) into (30) gives the coding gain of the proposed scheme as

$$
C(\mathrm{~dB})=\frac{-10 \cdot \log \left[\frac{1}{2}\left(\frac{\beta}{\alpha+\beta}\right)^{N-1}\right]}{N} .
$$

It is interesting to observe that, for symmetric channel configuration with $\alpha=\beta$, the coding gain is $3 \mathrm{~dB}$.

\section{Average Packet Delay}

We have shown that, when $\bar{\gamma} \rightarrow \infty$, the buffer states can only be in either $S^{(0)}$ or $S^{(1)}$, or a buffer can only be empty or contains one packet. When all buffers are empty, a new packet is transmitted to a relay with probability of $1 / N$. Further from (21) that $P\left(S^{(1)}\right)=1 / 2$, the probability that $Q_{k}$ contains one packet is given by

$$
P\left(Q_{k}=1\right)=P\left(S^{(1)}\right) \cdot \frac{1}{N}=\frac{1}{2 N} .
$$

Thus, when $\bar{\gamma} \rightarrow \infty$, the average buffer length at relay $R_{k}$ is given by

$$
\lim _{\bar{\gamma} \rightarrow \infty} \bar{L}_{k}=1 \cdot P\left(Q_{k}=1\right)=P\left(S^{(1)}\right) \cdot \frac{1}{N}=\frac{1}{2 N} .
$$

TABLE I

ASYMPTOTIC PERFORMANCE COMPARISON AMONG

DIFFERENT SCHEMES FOR SYMMETRIC CHANNELS

\begin{tabular}{|c|c|c|c|c|}
\hline & max-min & max-max & max-link & proposed \\
\hline diversity order & $N$ & $N$ & {$[N, 2 N)$} & $(N, N+1)$ \\
\hline coding gain & $0 \mathrm{~dB}$ & $3 \mathrm{~dB}$ & $3 \mathrm{~dB}$ & $3 \mathrm{~dB}$ \\
\hline average delay & 2 & $\frac{N L}{2}+1$ & $N L+1$ & 2 \\
\hline
\end{tabular}

From (16), and noticing that $\lim _{\bar{\gamma} \rightarrow \infty} P_{\text {out }}=0$, the average throughput at relay $Q_{k}$ is given by

$$
\lim _{\bar{\gamma} \rightarrow \infty} \eta_{k}=\frac{\lim _{\bar{\gamma} \rightarrow \infty}\left(1-P_{\text {out }}\right)}{2 N}=\frac{1}{2 N} .
$$

Finally, substituting (37) and (38) into (12), and further into (11), gives the average packet delay for $\bar{\gamma} \rightarrow \infty$ as

$$
\lim _{\bar{\gamma} \rightarrow \infty} \bar{D}=1+\frac{\frac{1}{(2 N)}}{\frac{1}{(2 N)}}=2 .
$$

It is clearly shown in (39) that, when SNR is high enough, the average packet delay of the proposed scheme is the same as that for the non-buffer-aided schemes.

\section{E. Comparison Between Different Schemes in Symmetric Channel Configuration}

For the symmetric channel configuration, Table I compares the diversity order, coding gain, and average delay for the nonbuffer-aided max-min, the traditional buffer-aided max-max and max-link, and the proposed schemes.

Table I shows that all buffer-aided schemes have the 3-dB coding gain over the max-min scheme. The proposed link has slightly higher diversity order than the max-max scheme but lower diversity order than the max-link scheme. In either the max-max or max-link scheme, the average packet delay increases linearly with relay number $N$ and buffer size $L$. In the proposed scheme, when $\bar{\gamma} \rightarrow \infty$, the average delay is fixed at 2, which is the same as that for the non-buffer-aided max-min scheme.

For asymmetric channels, the comparison between schemes is not the same as that shown in Table I, and this will be discussed in the following section.

\section{Simulations AND Discussions}

This section verifies the proposed scheme with numerical simulations, where the results for previously described maxlink and non-buffer-aided max-min schemes are also shown for comparison. In the following simulation, the transmission rates in all schemes are set as $r_{t}=2 \mathrm{~b} / \mathrm{s} / \mathrm{Hz}$, and simulation results are obtained with 1000000 Monte Carlo runs. Particularly in the proposed scheme, the simulation results always well match the theoretical analysis.

\section{A. Symmetric Channel Configuration: $\bar{\gamma}_{s r}=\bar{\gamma}_{r d}$}

In the first simulation, we consider a symmetric channel scenario that the source-to-relay and relay-to-destination links have the same average channel SNRs. 


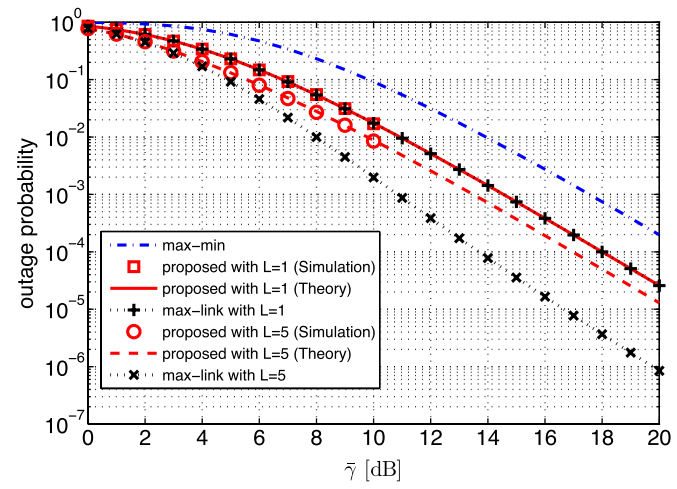

(a)

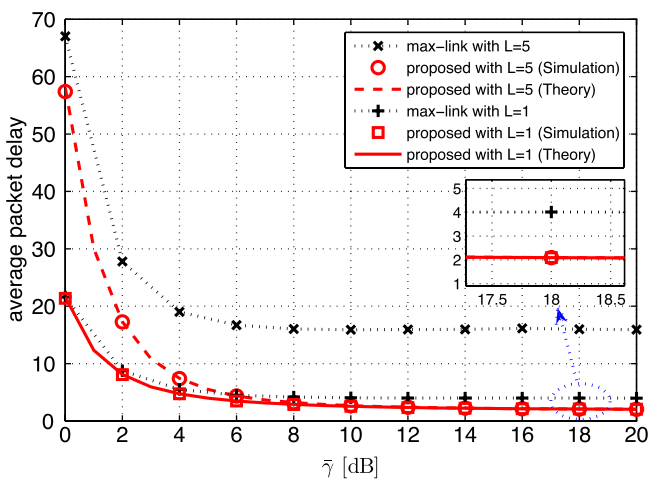

(b)

Fig. 2. (a) Outage probabilities and (b) average delay among different schemes, where $\bar{\gamma}_{s r}=\bar{\gamma}_{r d}=10 \mathrm{~dB}$.

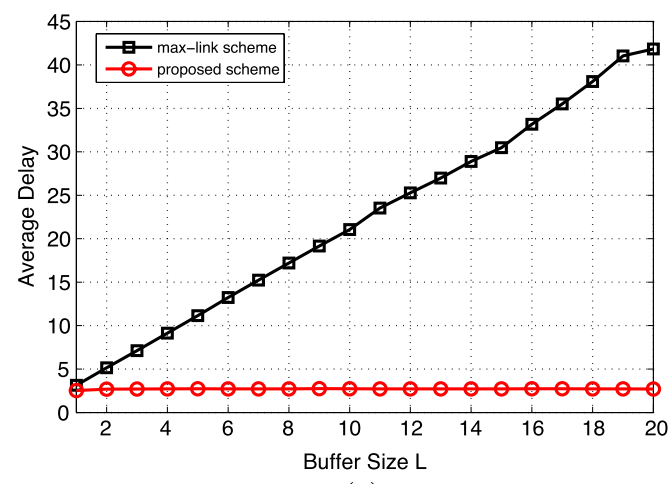

(a)

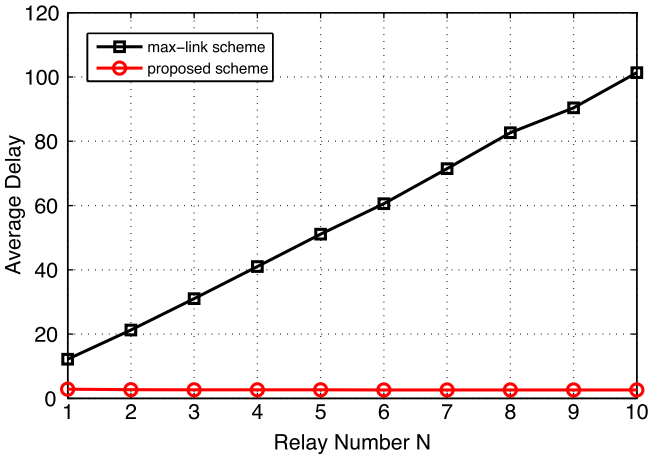

(b)

Fig. 3. Average packet delay comparison between the max-link and proposed schemes. (a) Delay versus buffer size, where $N=2$. (b) Delay versus relay number, where $L=10$.

Fig. 2(a) and (b) compares the outage probabilities and average packet delays for the non-buffered max-min, traditional max-link, and proposed schemes, respectively, where the relay number is fixed at $N=3$, and we let $\alpha=\beta=1.5$ and $\bar{\gamma}=$ $10 \mathrm{~dB}$ in (18), so that $\bar{\gamma}_{s r}=\bar{\gamma}_{r d}=15 \mathrm{~dB}$. Fig. 2(a) shows that, when the buffer size $L=1$, the proposed and max-link schemes have the same outage probabilities, where both have significantly better outage performance than the traditional nonbuffer-aided max-min scheme because of the 3-dB coding gain. When the buffer size increases to $L=5$, the proposed scheme has slightly better outage performance than that for $L=1$. This well matches the asymptotic analysis that, when $L \geq 2$, the diversity order is larger than $N$ but smaller than $(N+1)$ for the proposed scheme. On the other hand, for the max-link scheme, the outage performance improves more significantly with larger buffer size. This is because the diversity order of the max-link scheme goes up with the buffer size, until it reaches $2 N$ when $L \rightarrow \infty$. Fig. 2(b) shows that, even for $L=1$, the average delay of the max-link scheme is at least twice as much as that for the proposed scheme. When the buffer size increases to $L=5$, the average packet delay of the proposed scheme still maintains at 2 in high-SNR ranges, which is the same as that for $L=1$. On the other hand, when $L=5$, the average packet delay of the max-link scheme increases to 18 at high SNRs, which is nine times larger than that of the proposed scheme.

To further compare the delay performance of the max-link and proposed schemes in symmetric channels, Fig. 3(a) and (b) shows the average packet delay versus the buffer size and relay

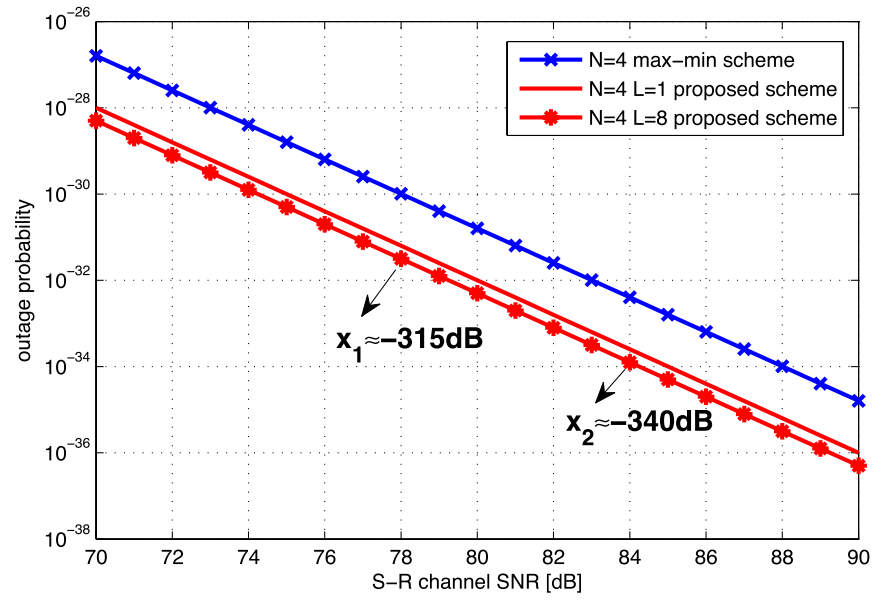

Fig. 4. Diversity order and coding gain of the proposed scheme.

number, respectively, where the average channel SNRs in both schemes are set as $10 \mathrm{~dB}$. In Fig. 3(a), the relay number is fixed at $N=2$, and the buffer size varies from 1 to 20. In Fig. 3(b), the buffer size is fixed at $L=10$, but the relay number varies from 1 to 10. It is clearly shown in both Fig. 3(a) and (b) that the average packet delay for the proposed scheme remains at a constant value of 2 . On the other hand, the packet delay in the max-link scheme goes up linearly with either $N$ or $L$.

To reveal the diversity order and coding gain of the proposed scheme, Fig. 4 compares the outage probabilities of the proposed and non-buffer-aided max-min schemes at very high 


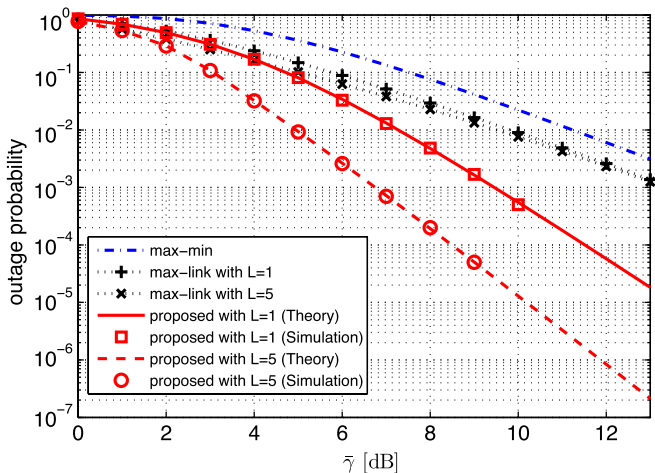

(a)

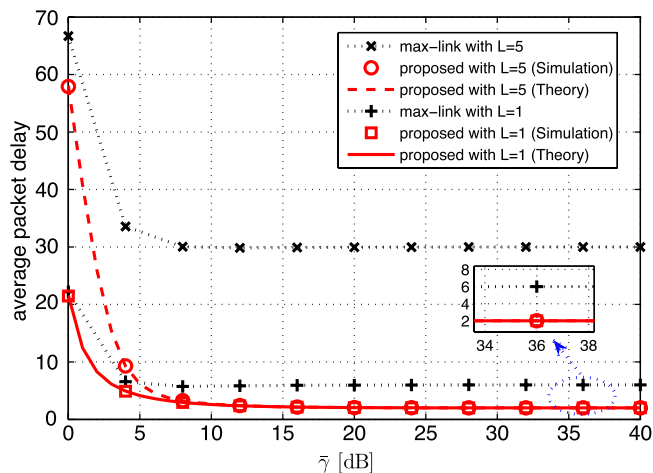

(b)

Fig. 5. (a) Outage probabilities and (b) average delay among different schemes, where $\bar{\gamma}_{s r}=20 \mathrm{~dB}$ and $\bar{\gamma}_{r d}=10 \mathrm{~dB}$.

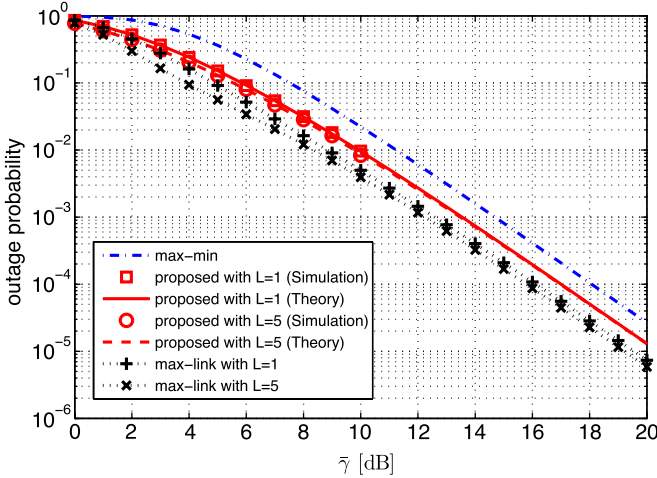

(a)

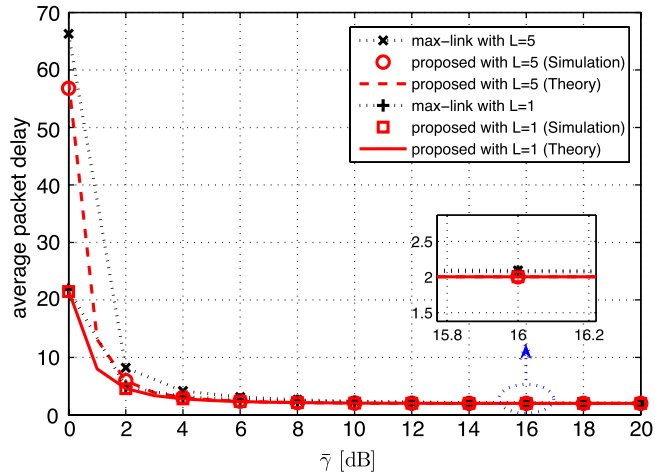

(b)

Fig. 6. (a) Outage probabilities and (b) average delay among different schemes, where $\bar{\gamma}_{s r}=10 \mathrm{~dB}$ and $\bar{\gamma}_{r d}=20 \mathrm{~dB}$.

SNRs, where the relay number is set as $N=4$ and all results are from theoretical analysis. First, the coding gain is clearly $3 \mathrm{~dB}$, by comparing the max-min and proposed schemes with $L=1$. For example, to achieve the outage probability of $10^{-34}$, the SNRs for the max-min and proposed schemes with $L=1$ are about 85 and $88 \mathrm{~dB}$, respectively. The diversity order of the proposed scheme is also clearly shown to be $(N, N+1)$ for $L \geq 2$. For example, as illustrated in the figure, for the proposed scheme with $L=8$, the SNRs to achieve the outage probabilities of -315 and $-340 \mathrm{~dB}$ are about 78 and $84 \mathrm{~dB}$, respectively. Then, according to the diversity order definition in (25), the diversity order is obtained as $(340-315) /(84-$ $78)=4.17$, which is clearly between $N=4$ and $N+1=5$.

\section{B. Asymmetric Channel Configuration: $\bar{\gamma}_{s r}>\bar{\gamma}_{r d}$}

In Fig. 5, we consider the asymmetric channel assumption that source-to-relay links are stronger than relay-to-destination links in average, where we let $\alpha=2, \beta=1$, and $\bar{\gamma}=10 \mathrm{~dB}$ in (18), so that $\bar{\gamma}_{s r}=20 \mathrm{~dB}$ and $\bar{\gamma}_{r d}=10 \mathrm{~dB}$, and relay number is fixed at $N=3$.

It is very interesting to observe in Fig. 5(a) that, for both $L=1$ and $L=5$, the outage performance of the proposed scheme is significantly better than the max-link scheme. This is because, when the source-to-relay links are stronger than the relay-to-destination links, the max-link scheme is more likely to select the source-to-relay links, so that the buffers are more likely full. This effectively decreases the number of available source-to-relay links, leading to lower diversity order. On the other hand, in the proposed scheme, while the channel condition gives higher priority to the source-to-relay selection, the selection rule gives higher priority to the relayto-destination link selection. This leads to a more "balanced" buffers at the relays, or fewer full or empty buffers, which again increases the diversity order.

Fig. 5(b) shows the average delay of the max-link even worse than that in symmetric channels. This is because the buffers are more likely to be full, or because of higher queuing length at buffers. On the contrary, the average delay for the proposed scheme is still as low as about 2 at high SNR.

Therefore, when $\bar{\gamma}_{s r}>\bar{\gamma}_{r d}$, the proposed scheme has better performance in both outage probability and average delay than the max-link scheme.

\section{Asymmetric Channel Configuration: $\bar{\gamma}_{s r}<\bar{\gamma}_{r d}$}

Fig. 6 assumes that the source-to-relay link is weaker than the relay-to-destination link in average, where we let $\alpha=1, \beta=2$, and $\bar{\gamma}=10 \mathrm{~dB}$ in (18), so that $\bar{\gamma}_{s r}=10 \mathrm{~dB}$ and $\bar{\gamma}_{r d}=20 \mathrm{~dB}$, and relay number is set as $N=3$. 
It is interesting to observe in Fig. 6 that, the max-link and proposed schemes have similar performance in both outage and average delay. This is because stronger relay-to-destination links "naturally" give higher priority to select the relay-todestination links. However, even under this channel assumption, the average packet delay is still better constrained in the proposed scheme than in the max-link scheme, particularly in low-SNR ranges.

\section{CONCLUSION}

This paper has proposed a novel buffer-aided relay selection scheme with significantly reduced packet delays. We have shown the outage and average delay performance under different channel configurations. To be specific, for symmetric $S \rightarrow R$ and $R \rightarrow D$ channels, the max-link scheme has better outage performance than the proposed. However, when $S \rightarrow R$ links are stronger, the proposed scheme performs better in outage than the max-link. On the other hand, when $R \rightarrow D$ links are stronger, the max-link and proposed schemes have similar outage performance. Therefore, if the relay nodes are evenly spread within an area as in many practical systems, it is reasonable to expect that the outage performances of the proposed and max-link schemes are similar. This will be left for future study. We also highlight that, in all cases, the proposed scheme has significantly better outage performance than the non-buffer-aided schemes, making it an attractive scheme in practical applications.

\section{ACKNOWLEDGMENT}

The authors would like to thank associate editor and anonymous reviewers for significantly improving the manuscript, particularly one of the reviewers for pointing out the parallel work in the Master's dissertation [19], in which Algorithms 2 and 3 were proposed with similar ideas to that in this paper. Algorithm 2 (which is based on giving higher priority to relays with longer queuing length) has similar performance to our proposed scheme, but it is more complicated to implement. Algorithm 3 trades off between outage and delay performance and, in some cases, may have longer delay than the "standard" buffer-aided max-link scheme. Our work was done independently from the work in the Master's dissertation.

\section{REFERENCES}

[1] J. N. Laneman, D. N. C. Tse, and G. W. Wornell, "Cooperative diversity in wireless networks: Efficient protocols and outage behavior," IEEE Trans. Inf. Theory, vol. 50, no. 12, pp. 3062-3080, Dec. 2004.

[2] Z. Ding, Y. Gong, T. Ratnarajah, and C. Cowan, "On the performance of opportunistic cooperative wireless networks," IEEE Trans. Commun., vol. 56, no. 8, pp. 1236-1240, Aug. 2008.

[3] B. Barua, H. Q. Ngo, and H. Shin, "On the SEP of cooperative diversity with opportunistic relaying," IEEE Commun. Lett., vol. 12, no. 10, pp. 727-729, Oct. 2008.

[4] G. Chen and J. A. Chambers, "Exact outage probability analysis for cooperative AF relay network with relay selection in presence of inter-cell interference," Electron. Lett., vol. 48, pp. 1346-1347, Oct. 2012.
[5] G. Chen, Y. Gong, and J. Chambers, "Study of relay selection in a multicell cognitive network," IEEE Wireless Commun. Lett., vol. 2, no. 4, pp. 435-438, Aug. 2013.

[6] A. Bletsas, A. Khisti, D. P. Reed, and A. Lippman, "A simple cooperative diversity method based on network path selection," IEEE J. Sel. Areas Commun., vol. 24, no. 3, pp. 659-672, Mar. 2006.

[7] A. Ikhlef, D. S. Michalopoulos, and R. Schober, "Max-max relay selection for relays with buffers," IEEE Trans. Wireless Commun., vol. 11, no. 3, pp. 1124-1135, May 2012.

[8] I. Krikidis, T. Charalambous, and J. S. Thompson, "Buffer-aided relay selection for cooperative diversity systems without delay constraints," IEEE Trans. Wireless Commun., vol. 11, no. 5, pp. 1957-1967, May 2012.

[9] Z. Tian, G. Chen, Y. Gong, Z. Chen, and J. A. Chambers, "Buffer-aided max-link relay selection in amplify-and-forward cooperative networks," IEEE Trans. Veh. Technol., vol. 64, no. 2, pp. 553-565, Feb. 2015.

[10] Z. Tian, Y. Gong, G. Chen, Z. Chen, and J. Chambers, "Buffer-aided link selection with network-coding in multi-hop networks," IEEE Trans. Veh. Technol., vol. 65, no. 9, pp. 7195-7206, Sep. 2016.

[11] N. Zlatanov, R. Schober, and P. Popovski, "Buffer-aided relaying with adaptive link selection," IEEE J. Sel. Areas Commun., vol. 31, no. 8, pp. 1530-1542, Aug. 2013.

[12] N. Zlatanov, R. Schober, and P. Popovski, "Buffer-aided relaying with adaptive link selection-fixed and mixed rate transmission," IEEE Trans. Inf. Theory, vol. 59, no. 5, pp. 2816-2840, May 2013.

[13] G. Chen, Z. Tian, Y. Gong, and J. A. Chambers, "Decode-and-forward buffer-aided relay selection in cognitive relay networks," IEEE Trans. Veh. Technol., vol. 63, no. 9, pp. 4723-4728, Mar. 2014.

[14] G. Chen, Z. Tian, Y. Gong, Z. Chen, and J. A. Chambers, "Max-ratio relay selection in secure buffer-aided cooperative wireless networks," IEEE Trans. Inf. Forensics Security, vol. 9, no. 4, pp. 719-729, Apr. 2014.

[15] J. R. Norris, Markov Chains. Cambridge, U.K.: Cambridge Univ. Press, 1998.

[16] J. D. C. Little, "A proof of the queueing formula: $L=\lambda \omega$," Oper. Res., vol. 9, no. 3, pp. 383-388, 1961.

[17] R. Narasimhan, "Throughput-delay performance of half-duplex hybridARQ relay channels," in Proc. IEEE ICC, Beijing, China, May 19-23, 2008, pp. 986-990.

[18] G. Chen, Y. Gong, P. Xiao, and T. Rahim, "Dual antenna selection in selfbackhauling multiple small cell networks," IEEE Commun. Lett., vol. 20, no. 8, pp. 1611-1614, Aug. 2016.

[19] D. Poulimeneas, "Back-pressure-like mechanisms on relay selection policies for cooperative diversity systems," M.S. thesis, Dept. Electr. Eng., KTH Roy. Inst. Technol., Stockholm, Sweden, 2015. [Online]. Available: http://kth.diva-portal.org/smash/record.jsf?pid=diva2\%3A813435 \&dswid $=537 \% 2$

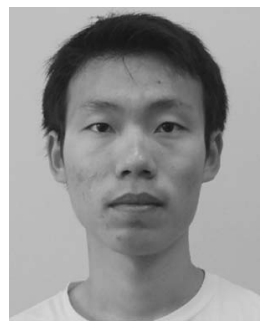

Zhao Tian (S'12-M'15) received the B.Eng. degree and the Ph.D. degree in electronic, electrical and systems engineering from Loughborough University, Loughborough, U.K., in 2012 and 2015, respectively.

$\mathrm{He}$ is currently a Knowledge Transfer Project Associate in Computer Science with Aston University, Birmingham, U.K., and IGI Ltd., Birmingham. His current research interests include the general field of wireless communications, with emphasis on bufferaided relaying, machine learning, and data mining.

Dr. Tian was a recipient of the Full Postgraduate Scholarship from the Engineering and Physical Sciences Research Council while working toward the Ph.D. degree. 


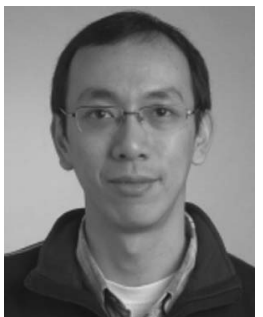

Yu Gong received the B.Eng. and M.Eng. degrees in electronic engineering from the University of Electronics and Science Technology of China, Chengdu, China, in 1992 and 1995, respectively, and the Ph.D. degree in communications from the National University of Singapore, Singapore, in 2002.

After Ph.D. graduation, he took several research positions with the Institute of Infocomm Research, Singapore, and also with Queen's University of Belfast, Belfast, U.K. From 2006 and 2012, he was an Academic Member with the School of Systems Engineering, University of Reading, Reading, U.K. He has been with the School of Electronic, Electrical and Systems Engineering, Loughborough University, Loughborough, U.K., since July 2012. His research interests are in the area of signal processing and communications, including wireless communications, cooperative networks, nonlinear and nonstationary system identification, and adaptive filters.

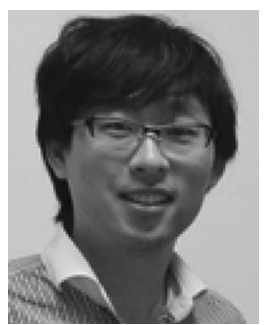

Gaojie Chen (S'09-M'12) received the B.Eng. and B.Ec. degrees in electrical information engineering and international economics and trade from the Northwest University, Shaanxi, China, in 2006 and the M.Sc. (Distinction) and Ph.D. degrees in electrical and electronic engineering from Loughborough University, Loughborough, U.K., in 2008 and 2012, respectively.

From 2008 to 2009, he was a Software Engineering with DTmobile, Beijing, China, and from 2012 to 2013, he was a Research Associate with the School of Electronic, Electrical and Systems Engineering, Loughborough University, Loughborough, U.K. From 2014 to 2015, he was a Research Fellow with the 5G Innovation Centre, Faculty of Engineering and Physical Sciences, University of Surrey, Surrey, U.K. He is currently a Research Associate with the Department of Engineering Science, University of Oxford, Oxford, U.K. His current research interests include information theory, wireless communications, cooperative communications, cognitive radio, secrecy communication, and random geometric networks.

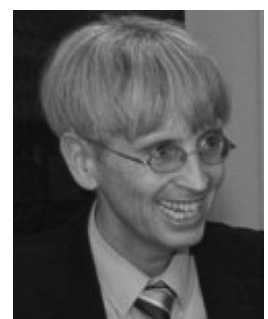

Jonathon A. Chambers (S'83-M'90-SM'98-F'11) received the $\mathrm{Ph} . \mathrm{D}$. and D.Sc. degrees in signal processing from the Imperial College of Science, Technology and Medicine (Imperial College London), London, U.K., in 1990 and 2014, respectively.

From 1991 to 1994, he was a Research Scientist with the Schlumberger Cambridge Research Centre, Cambridge, U.K. In 1994, he returned to Imperial College London as a Lecturer in signal processing and was promoted to Reader (Associate Professor) in 1998. From 2001 to 2004, he was the Director of the Centre for Digital Signal Processing and a Professor in signal processing with the Division of Engineering, King's College London. From 2004 to 2007 , he was a Cardiff Professorial Research Fellow with the School of Engineering, Cardiff University, Cardiff, U.K. In 2007-2014, he led the Advanced Signal Processing Group, the School of Electronic, Electrical and Systems Engineering, Loughborough University, Loughborough, where he is now a Visiting Professor. In 2015, he joined the School of Electrical and Electronic Engineering, Newcastle University, Newcastle upon Tyne, U.K., where he is a Professor in signal and information processing and heads the Communications, Sensors, Signal and Information Processing Research Group $\left(\mathrm{ComS}^{2} \mathrm{IP}\right)$. He is also a Guest Professor with Harbin Engineering University, Harbin, China. He is a coauthor of the books "Recurrent Neural Networks for Prediction: Learning Algorithms, Architectures and Stability" (New York, NY, USA: Wiley, 2001) and "EEG Signal Processing" (New York, NY, USA: Wiley, 2007). He has advised more than 60 researchers through to Ph.D. graduation and published more than 400 conference proceedings and journal articles, many of which are in IEEE journals. His research interests include adaptive and blind signal processing and their applications.

Dr. Chambers is a Fellow of the Royal Academy of Engineering, U.K., and the Institution of Electrical Engineers. He was the Technical Program Chair of the 15th International Conference on Digital Signal Processing and the 2009 IEEE Workshop on Statistical Signal Processing, both held in Cardiff, U.K., and a Technical Program Cochair of the 36th IEEE International Conference on Acoustics, Speech, and Signal Processing, Prague, Czech Republic. He received the first QinetiQ Visiting Fellowship in 2007, for his outstanding contributions to adaptive signal processing and his contributions to QinetiQ, as a result of his successful industrial collaboration with the international defense systems company QinetiQ. He has served on the IEEE Signal Processing Theory and Methods Technical Committee for six years and the IEEE Signal Processing Society Awards Board for three years. He is currently a member of the IEEE Signal Processing Conference Board and the European Signal Processing Society Best Paper Awards Selection Panel. He has also served as an Associate Editor for the IEEE TRANS ACTIONS ON SignAL PRoCESSING for three terms over the periods 1997-1999 and 2004-2007 and since 2011 as a Senior Area Editor. 